\title{
Effect of aluminum and fluoride on R2R3-MYB transcription factor characterization and expression in Camellia sinensis
}

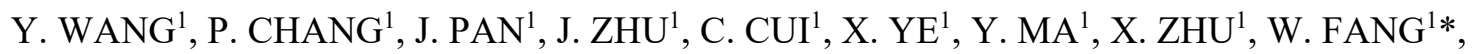 \\ and C. JIANG ${ }^{2 *}$ \\ College of Horticulture, Nanjing Agricultural University, Nanjing 210095, P.R.China ${ }^{1}$ \\ State Key Laboratory of Tea Plant Biology and Utilization, Anhui Agricultural University, Hefei 230036, P.R.China ${ }^{2}$
}

\begin{abstract}
Tea plant (Camellia sinensis) is a typical plant that accumulates abundant aluminum $(\mathrm{Al})$ and fluoride $(\mathrm{F})$. $\mathrm{Al}$ and $\mathrm{F}$ play vital roles in the growth and development in tea plant. MYB (myeloblastosis) is one of the largest transcription factor families in plant, which plays an important role in the stress responses. As the largest and multifunctional subfamily of MYB, R2R3-MYB subfamily participates in multiple abiotic stresses in plant. However, there are few studies about R2R3-MYB in tea plant, especially in the accumulation of $\mathrm{Al}$ and $\mathrm{F}$ during its growth. Here, we identified 43 CsMYB genes from the transcriptome database of tea plant and analyzed the relative expression in $\mathrm{Al}$ and $\mathrm{F}$ treatments. According to the Arabidopsis thaliana classification, 43 CsMYB genes are divided into 18 subgroups via phylogenetic tree analysis. All 43 CsMYB proteins have the typical R2R3-MYB domain by MEME prediction. Moreover, 9 CsMYB genes (CsMYB11, CsMYB17, CsMYB29, CsMYB38 to CsMYB43) that related to abiotic stress were selected from 43 CsMYB genes for a further study, and the relative expressions showed that they are tissue-specifically expressed. In addition, the results of different concentrations of Al treatments showed that CsMYB11, -17, -29, -38 and -41 are stimulated by $\mathrm{Al}$ treatment. The results of different concentrations of $\mathrm{F}$ treatments showed that the CsMYB11, $-17,-38,-39,-40,-41,-42$ and -43 participate in $\mathrm{F}$ accumulation of $C$. sinensis roots. Our research establishes a solid foundation for further investigation into the molecular functions of R2R3-MYB transcription factors in C. sinensis.
\end{abstract}

Additional key words: Arabidopsis thaliana, phylogenetic tree, stress response, tea growth and development.

\section{Introduction}

Tea plant [Camellia sinensis (L.) O. Kuntze] is a leaf-type crop. It is known to be a strong accumulator of aluminum (Mukhopadyay et al. 2012). Under acidic conditions, $\mathrm{Al}$ is accumulated in tea plant mainly in leaves, especially in old leaves up to $5.6 \mathrm{~g}(\mathrm{Al}) \mathrm{kg}^{-1}$ (f.m.) and less in young leaves $1 \mathrm{~g}(\mathrm{Al}) \mathrm{kg}^{-1}$ (f.m.) (Wong et al. 1998). Tea leaves take up $\mathrm{Al}$ during growth and contain up to $30 \mathrm{~g}(\mathrm{Al}) \mathrm{kg}^{-1}$ (d.m.) (Tolra et al. 2011). However, tea plants with high Al content do not exhibit symptoms of toxicity, rather growth stimulation (Hajiboland et al. 2013). Previous studies have showed that the tea plant physiological characteristics and metabolites are changed in response to $\mathrm{Al}$ (Hajiboland et al. 2013, Xu et al. 2016). Xu et al. (2017) also find 755 and 1059 differentially expressed proteins in tea roots and leaves under $\mathrm{Al}$ stress, respectively. These results enhance understanding of the stimulatory effects of $\mathrm{Al}$ on tea plant. Recent study has revealed that the myeloblastosis (MYB) transcription factor (TF) families are involved in response of tea roots to high $\mathrm{Al}$ content ( $\mathrm{Li}$ et al. 2017). Nonetheless, the molecular mechanism of tea plants response to $\mathrm{Al}$ is still unclear.

Tea plant is often assumed to be highly tolerant to fluoride (F) as well, as they can accumulate a lot of $\mathrm{F}$ without showing symptoms of toxicity (Ruan and Wong

Submitted 18 April 2018, last revision 29 August 2018, accepted 14 September 2018.

Abbreviations: DBD - DNA binding domain; MYB - myeloblastosis; RT-qPCR - reverse transcription quantitative polymerase chain reaction; $\mathrm{TF}$ - transcription factor.

Acknowledgements: This work was supported by the National Natural Science Foundation of China (No. 31770733), the Opening Project of State Key Laboratory of Tea Plant Biology and Utilization (No. SKLTOF20150113), the Fundamental Research Funds For the Central Universities (No. KYZ201509), the earmarked fund for the China Agriculture Research System (CARS-19) and the Modern Agriculture Industry System in Jiangsu Province (SXGC[2017]279).

* Corresponding authors; faxes: (+86)-25-84395182 and (+86)-0551-5786982, e-mails: fangwp@njau.edu.cn; jiangcj@ahau.edu.cn 
2001). Fluorides are accumulated mainly in leaves and increased with age of tea leaves. The highest F content is found in fallen leaves, followed by mature leaves, young leaves, branches, and roots (Fung et al. 1999). Meanwhile, high $\mathrm{F}$ treatment can influence photosynthesis, antioxidant defense, and cell ultrastructure of tea leaves (Cai et al. 2016). Previous reports demonstrated that F is taken up by tea roots through active rather than passive processes (Zhang et al. 2013). In addition, the F uptake of tea plant is affected by many environmental factors, such as the $\mathrm{pH}$ and other mineral elements $\mathrm{Ca}^{2+}$ and $\mathrm{Al}^{3+}$ (Ruan et al. 2004, Yang et al. 2016). Recent studies have also found that many genes of tea plant are involved in the absorption of F ( $\mathrm{Li}$ et al. 2017). However, little is known about the molecular mechanisms of $\mathrm{F}$ accumulation in tea plant.

The MYB TF family exists widely in eukaryotes and it is one of the largest and most diverse families of TF in the plant kingdom (Riechmann et al. 2000). The distinguishing property of MYB proteins is a highly conserved MYB domain consisting of 1 - 4 imperfect tandem repeats (MYB-repeat) at the N-terminus. The MYB repeat is 50 - 53 amino acids in length and contains three regularly distributed tryptophan (or phenylalanine) residues. Each MYB-repeat encodes three $\alpha$-helices, with the second and third helices forming a helix-turn-helix structure, which recognizes and binds to the DNA major groove at the specific recognition site C/TAACG/TG (Stracke et al. 2001). The MYB family is divided into different types according to the number of MYB-repeats: 4R-MYB, 3R-MYB (R1R2R3-MYB), 2R-MYB (R2R3-MYB), and 1R-MYB (R1/R2 MYB) type (Dubos et al. 2010). The 4R-MYB group is the smallest and contains four R1/R2-like repeats, it is rarely known of their functions in plants (Dubos et al. 2010). The 3R-MYB group is also small, and its constituents function in cell cycle control (Ito 2005, Haga et al. 2007). The 2R-type MYB genes constitute the largest plant subfamily and are thought to have evolved from the same ancestor as 3R-MYB genes (Zhou et al. 2015). The R2R3-MYB is one of the largest families of MYB TF, which shared an R2R3 domain with 104 amino acid residues, including five conserved tryptophan residues. More than over 100 R2R3-MYB members have been reported in dicots and monocots (Wilkins et al. 2009). Whole genome identification of R2R3-MYB has been carried out in different plant species. For example, 126 R2R3-MYB genes were found in Arabidopsis (Dubos et al. 2010), 192 in poplar (Wilkins et al. 2009), 157 in maize (Du et al. 2012a) and 244 in soybean (Du et al. 2012b). They have been implicated in multiple biological processes, such as biotic and abiotic stresses (Lippold et al. 2009, Segarra et al. 2009), determination of cell shape (Noda et al. 1994), and regulation of differentiation (Xie et al. 2010). For example, AtMYB59 regulates root development through the control of cell cycle progression at the root tips $(\mathrm{Mu}$ et al. 2009) and AtMYB77 regulates lateral root formation by modulating the expression of auxin-inducible genes (Shin et al. 2007). AtMYB68 (subgroup 14) is a root growth specific regulator, impacting overall plant development under unfavourable conditions (e.g. high temperature) (Feng et al. 2004). AtMYB96 has been shown to be involved in regulation of lateral root growth in response to drought stress via the ABA-auxin signalling network (Seo and Park 2009). Extensive studies in Arabidopsis have provided a better understanding of the R2R3-MYB gene family. In tea plant, the R2R3-MYB is found to be related to the flavonoid biosynthesis (Zhao et al. 2013). In addition, a recent study has showed that the purple foliage coloration of tea plant is activated by the R2R3-MYB (Sun et al. 2016). Whereas little is known about this family in tea plant under abiotic stresses, especially under Al and F stresses.

In this study, we screened and identified $43 C$. sinensis $R 2 R 3-M Y B$ genes, and analyzed the relative expression in different tissues and after $\mathrm{Al}$ and $\mathrm{F}$ treatments. The results of this study can reveal the structures and functions of the $C s M Y B$ genes and serve as a basis for future research to elucidate the regulatory mechanisms of R2R3-MYB transcription factor in tea plant.

fruits. All the samples were immediately frozen in liquid nitrogen and then stored at $-80{ }^{\circ} \mathrm{C}$ until required.

Plants and Al and $\mathbf{F}$ treatments: The two-year-old cutting seedlings of tea [Camellia sinensis (L.) O. Kuntze] cv. Longjingchangye were pre-incubated in control nutrient solution in a greenhouse under a temperature of $23{ }^{\circ} \mathrm{C}$, a $70 \%$ relative humidity, a $14-\mathrm{h}$ photoperiod, and an irradiance of $250-280 \mu \mathrm{mol} \mathrm{m} \mathrm{m}^{-2} \mathrm{~s}^{-1}$ for 2 weeks. Then tea plants were grown hydroponically in an incubator with 0 , 0.4 , and $2 \mathrm{mM} \mathrm{Al}^{3+}$ or 0,8 , and $16 \mathrm{mg} \mathrm{dm}^{-3} \mathrm{~F}^{-}$and growth and the uptake of $\mathrm{Al}^{3+}$ and $\mathrm{F}^{-}$were determined. Root samples were collected after $0,3,6,12$, and $24 \mathrm{~h}$. In addition, different tissues from conventionally grown tea plant were collected, including roots, stems, first and fourth leaves, buds, flowers, pollen and pollen tubes, and
Phylogenetic tree construction and sequence analysis of the CsMYB family: We searched for all the putative MYB proteins that retrieved from $C$. sinensis transcriptome data (Pan et al. 2016) and unpublished transcriptome data (NCBI accession: SRP149468). Using $B L A S T$ search of amino acid sequences, we detected whether the $C s M Y B$ s contain highly conserved DNA binding domain (DBD) or not. Then we determined the sequence similarity of the $C S M Y B$ s with Arabidopsis $M Y B s$ by $N C B I$. After excluding the $C s M Y B$ s that lack 
DBD, we finally identified the $43 C s M Y B$ s which can be used for further analysis. The MYB family of Arabidopsis was downloaded from the plant TF database (http://planttfdb.cbi.pku.edu.cn/). The amino acid sequences of MYB proteins from tea and Arabidopsis were aligned. Subsequently, we constructed an unrooted phylogenetic tree by using the neighbor-joining method and bootstrap analysis (1 000 replicates) in MEGA 6.0 (http:/www.megasoftware.net). Motif location was analyzed using MEME v. 4.10.1 (http://memesuite.org/tools/meme). With the following parameter settings: the distribution of motifs, 0 or 1 per sequence; maximum number of motifs to find 20; minimum width of motif 6; maximum width of motif 53. In addition, only motifs with an $e$-value $\leq 1 \times 10^{-10}$ were retained for further analysis.
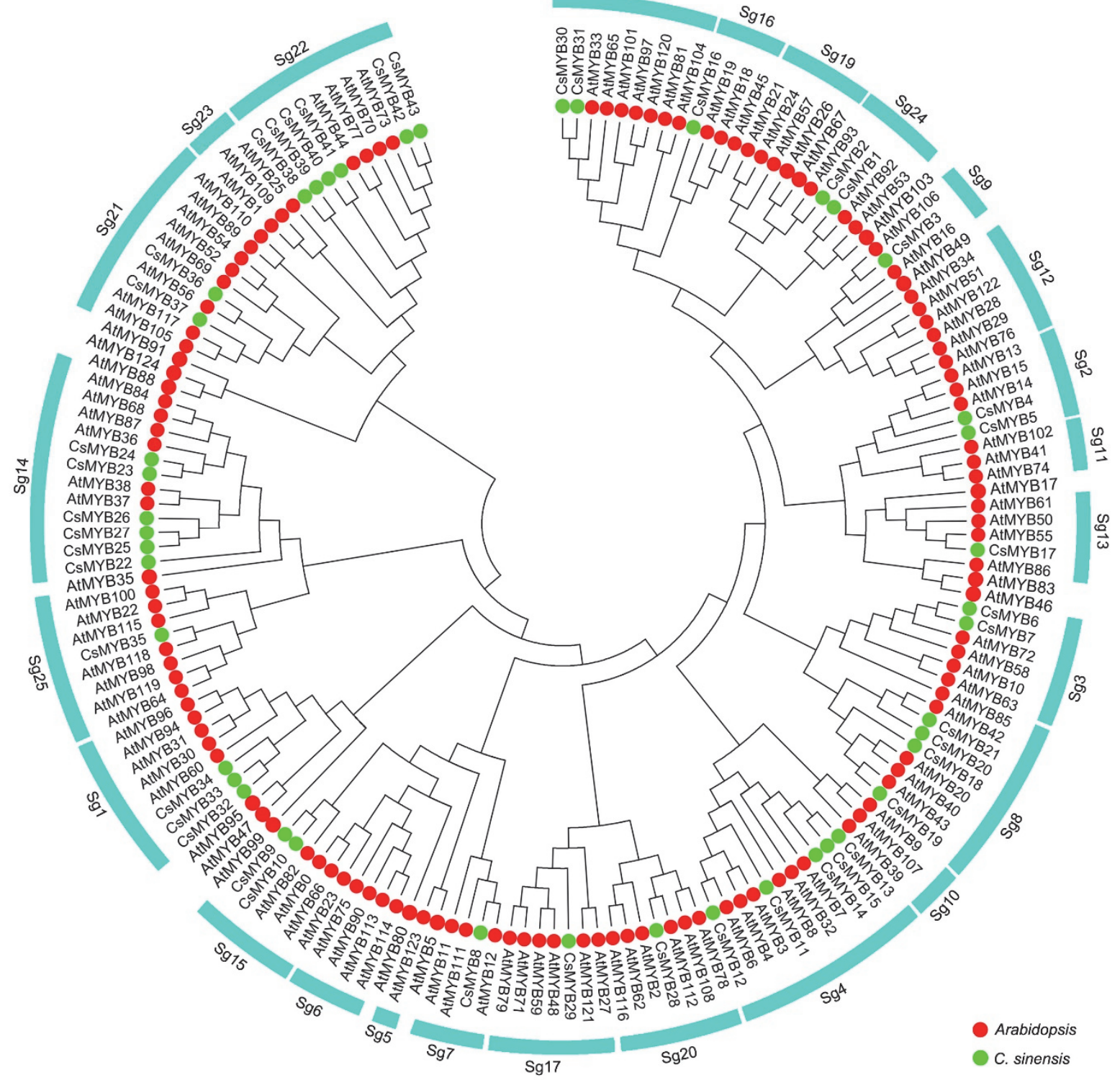

Fig. 1. The phylogenetic tree was constructed between tea plant and Arabidopsis. The sequences include 43 CsMYB proteins and 126 AtMYB proteins.

Reverse transcription quantitative PCR analysis: The total RNA was isolated from all samples by using the RNA isolation kit (Huayueyang, Beijing, China) in accordance with the manufacturer's instructions. First-strand cDNA was synthesized from samples RNA using the Prime
Script $^{\mathrm{TM}} \mathrm{RT}$ reagent kit (TaKaRa, Dalian, China) and then diluted 10-fold. The $C s \beta$-actin gene was selected as an internal reference to normalize the expression of the selected CsMYB genes. The sequences of all primers are listed in Table 1 Suppl. The profile of cycling was based 
on SYBR Premix Ex-Taq $\mathrm{kit}^{\circledR}$ (TaKaRa). The RT-qPCR was performed on a Bio-Rad (Hercules, USA) CFX96 platform under conditions: denaturation at $95^{\circ} \mathrm{C}$ for $30 \mathrm{~s}$; 40 cycles of $95^{\circ} \mathrm{C}$ for $5 \mathrm{~s}$ and $60^{\circ} \mathrm{C}$ for $30 \mathrm{~s}$; and then $95{ }^{\circ} \mathrm{C}$ for $10 \mathrm{~s}$; a final extension at $65^{\circ} \mathrm{C}$ for $5 \mathrm{~s}$ and then $95{ }^{\circ} \mathrm{C}$ for $5 \mathrm{~s}$. Each reaction was performed in a total volume of $20 \mathrm{~mm}^{3}$ containing $0.4 \mathrm{~mm}^{3}(10 \mathrm{mM})$ of each gene-specific primer, $2 \mathrm{~mm}^{3}$ of diluted cDNA as the template, $7.2 \mathrm{~mm}^{3}$ of $\mathrm{ddH}_{2} \mathrm{O}$, and $10 \mathrm{~mm}^{3}$ of $S Y B R$ Premix Ex Taq $(2 \times)$ mixture. Each independent cDNA sample was performed with three independent biological replications. We used the $2^{-\triangle \Delta C T}$ method to calculate the expressions of CsMYB genes relative to the Cs $\beta$-actin gene (Pfaffl 2001). The average of three $2^{-\Delta \Delta \mathrm{CT}}$ values obtained from the triplicate experiments showed the final expression.

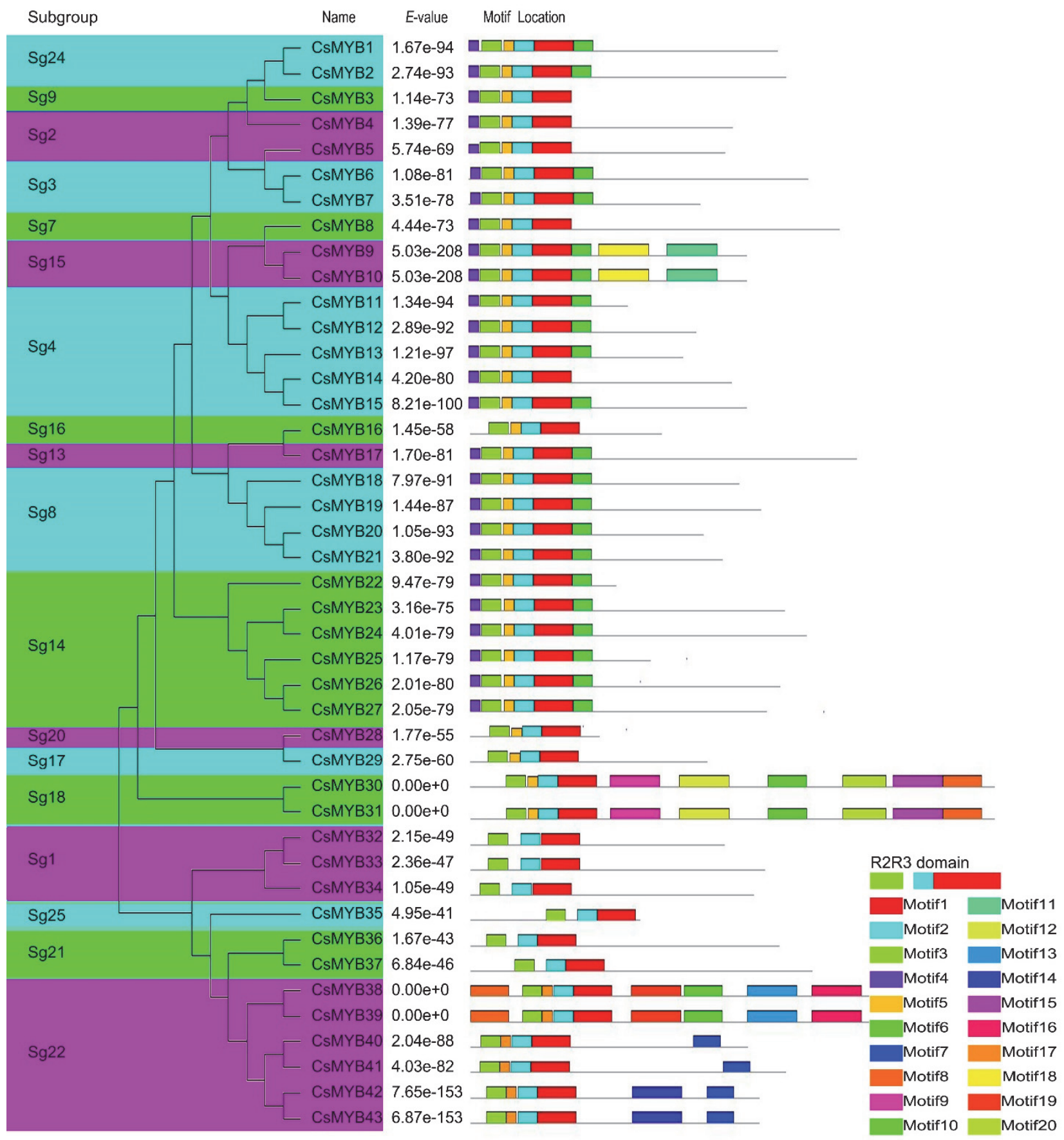

Fig. 2. The conserved motif of CsMYB proteins. The unrooted phylogenetic tree was constructed using the conserved domain sequences using MEGA. Motifs of R2R3-MYB proteins were identified by MEME. Each motif is represented by a colored box and numbered. 


\section{Results}

To identify R2R3-MYB family genes in tea plant, $B L A S T$ searches were carried out against tea plant genomes, including $C$. sinensis transcriptome data (Pan et al. 2016) and $N C B I$ data. Among the potential CsMYB genes, $43 C s M Y B$ genes encoding MYB domain from the transcriptome database of tea plant were selected for further investigation and their corresponding amino acid sequences were confirmed using NCBI BLAST program (Table 2 Suppl.). These genes were named CsMYBO1 to CsMYB43. In order to confirm the obtained classifications and to further categorize the $C s M Y B$ family, a phylogenetic tree based on the alignment of the 126 AtMYB proteins and 43 CsMYB proteins was constructed (Fig. 1). According to the classification of R2R3-MYB family genes in Arabidopsis, 43 CsMYB proteins in tea plant were further identified as belonging to 18 subgroups. Among the $C s M Y B$ subgroups, subgroups 7, 9, 13, 16, 17, 20, and 25 have one $C s M Y B$ gene; subgroups 2, 3, 15, 18, 21, and 24 have two $C s M Y B$ genes; subgroup 1 has three $C s M Y B$ genes; subgroup 8 has four CsMYB genes; subgroup 4 has five $C s M Y B$ genes; and subgroups 14 and 22 have six $C s M Y B$ genes. Given the above classification, the 43 CsMYB genes were subjected to further analyses.

To further reveal the diversification of $43 C s M Y B$ genes, 20 distinct conserved motifs were identified in
C. sinensis R2R3-MYB proteins using MEME (Fig. 2). All of the closely related members in the phylogenetic analysis had common conserved motifs among the R2R3-MYB proteins, which was consistent with those results of the presented phylogenetic analysis. In this study, all of the CsMYB genes have the typical R2R3-MYB domain (Fig. 3). In general, the basic regions of the R2 and R3 repeats had 50 basic residues. Highly conserved tryptophan residue was located in the 10, 30, and 50 positions of the $\mathrm{R} 2$ repeat, and the 31 and 50 positions of the R3 repeat. Motifs 1, 2, and 3 consist of the integrated R2R3-MYB domain. Motifs 4 and 5 were the shortest with 10 amino acids. The motif 4 was not observed in subgroups 1, $16-18,20-22$, and 25. Motif 5 was absent in subgroups 1, 21, and 25. Five CsMYB proteins belonged to subgroup 4, while only one protein (CsMYB14) had no motif 6 . Motifs 8 and 9 had the longest sequence of 49 amino acids, followed by motif 19. Motif 9 was only found in two CsMYB proteins (CsMYB30 and CsMYB31). Only subgroup 15 has motifs 11 and 12 . Motifs 13, 14, and 16 were not observed in subgroup 22. Six CsMYB proteins belonged to subgroup 22, while two CsMYB proteins (CsMYB 38 and CsMYB 39) had the same motifs. Motifs 15,17 , and 20 was absent in subgroup 18.

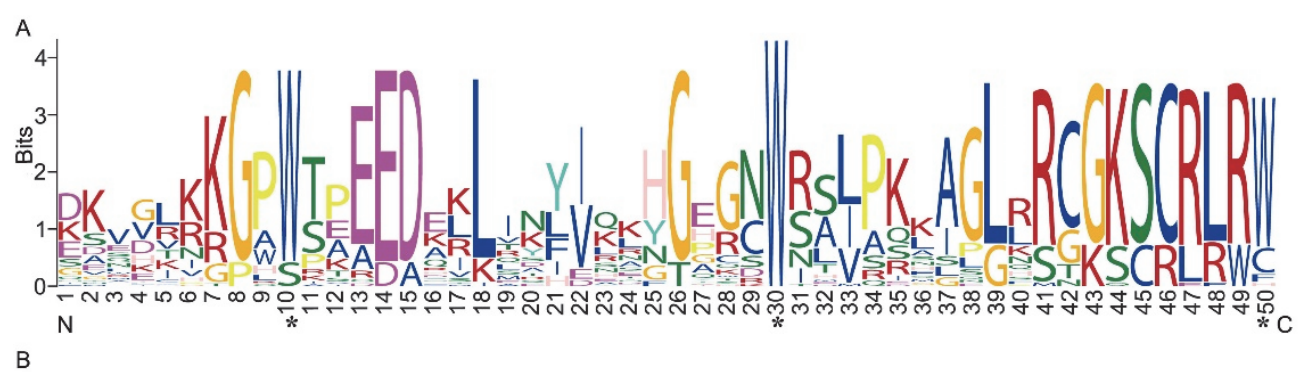

B

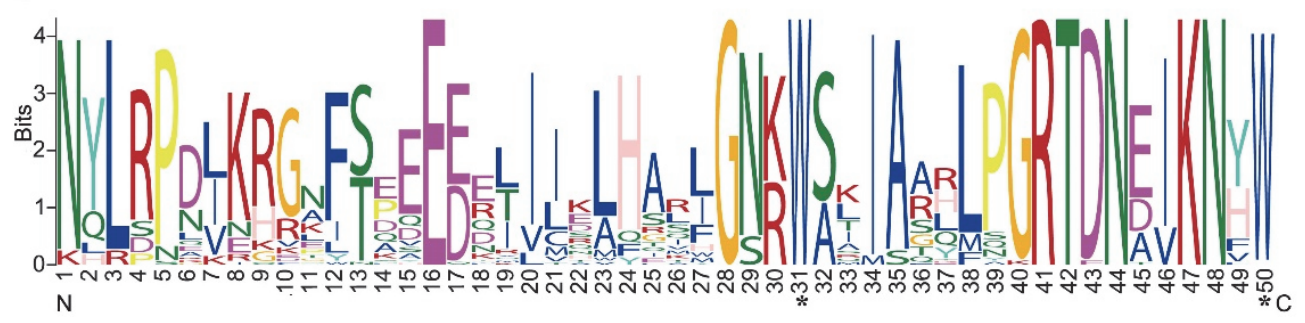

Fig. 3. The logos of R2 (A) and R3 (B) MYB repeats highly conserved across 43 R2R3 MYB proteins in Camlia sinensis. Asterisks represent the conserved tryptophan residues (Trp) in the MYB domain.

The RT-qPCR analysis was performed to elucidate the expression patterns of $C S M Y B$ genes in different tissues of C. sinensis (Fig. 4). The CsMYB genes showed a diverse expression patterns. For example, CsMYB11 (subgroup 4) and CsMYB40 (subgroup 22) displayed the highest expression in flowers and a relatively low expression in fruits. CSMYB17 (subgroup 13) revealed the highest expression in pollen and a relatively low expression in fruits. CsMYB29 (subgroup 17) and CsMYB38 (subgroup
22) are expressed strongly in roots. CsMYB39 (subgroup 22) showed the highest expression in stems and a relatively low expression in pollen. CsMYB41 (subgroup 22) and CSMYB42 (subgroup 22) revealed high expressions in pollen tubes. The expression of CSMYB43 (subgroup 22) was mostly observed in fruits and pollen tubes, whereas low expression was observed in other organs.

To detect the impact of $\mathrm{Al}$ and $\mathrm{F}$ on the expression of the nine CsMYB genes (CsMYB11, CsMYB17, CsMYB29, 
CsMYB38, and CsMYB40 to CsMYB43), tea plants were treated with a range of $\mathrm{Al}^{3+}$ and $\mathrm{F}^{-}$concentration. According to the preliminary experiments, the new roots of $C$. sinensis grew faster under $0.4 \mathrm{mM} \mathrm{Al}^{3+}$ than under
0 and $2 \mathrm{mM} \mathrm{Al}^{3+}$ treatment. The CsMYB11 (subgroup 4) was down-regulated under 0.4 and $2 \mathrm{mM} \mathrm{Al}^{3+}$. The expression of CSMYB17 (subgroup 13) was the highest after 3 and $12 \mathrm{~h}$ exposure to $0.4 \mathrm{mM} \mathrm{Al}^{3+}$. CsMYB17
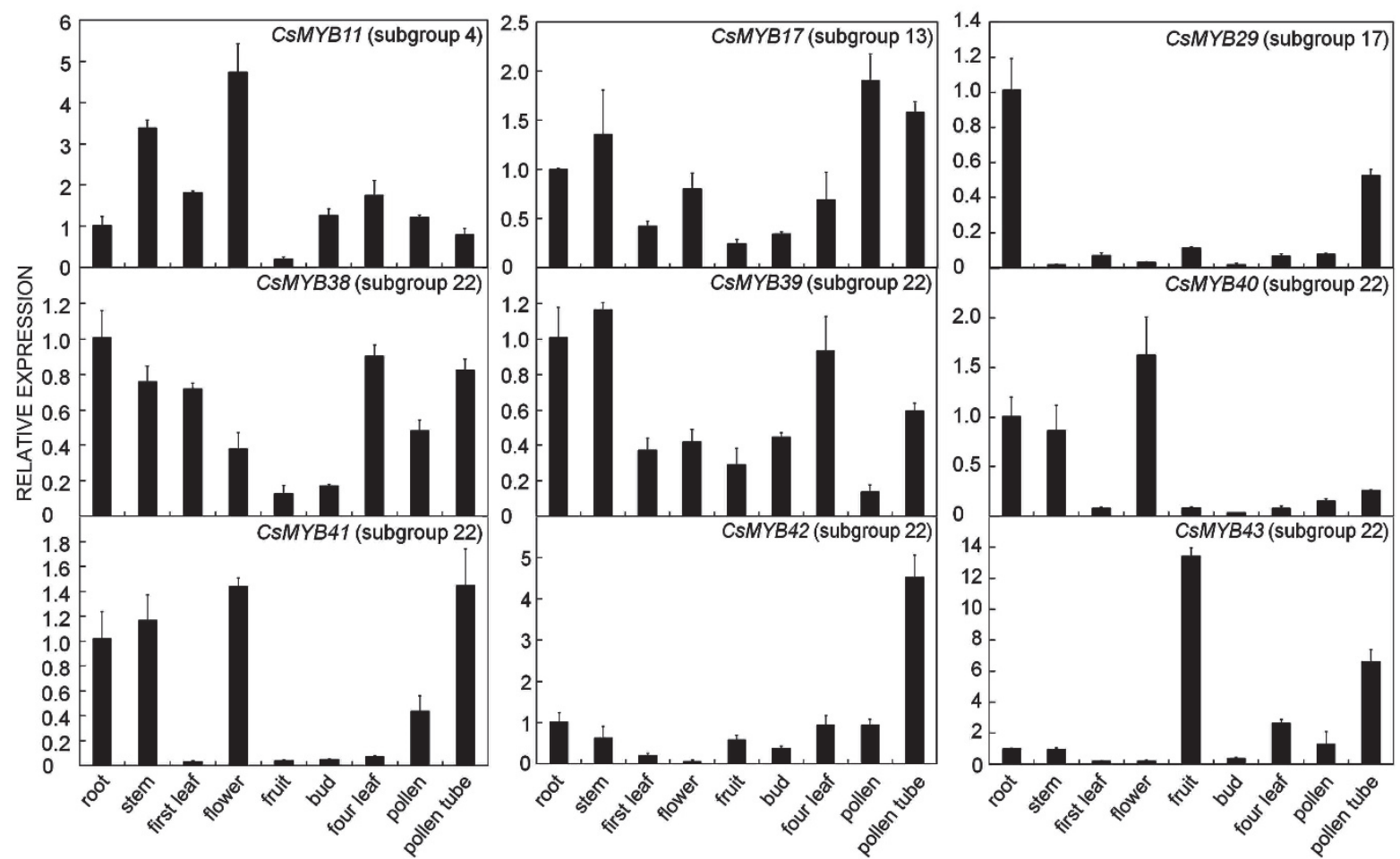

Fig. 4. The relative expressions of 9 CsMYB genes in different tissues (roots, stems, first leaves, flowers, fourth leaves, pollen, pollen tubes).
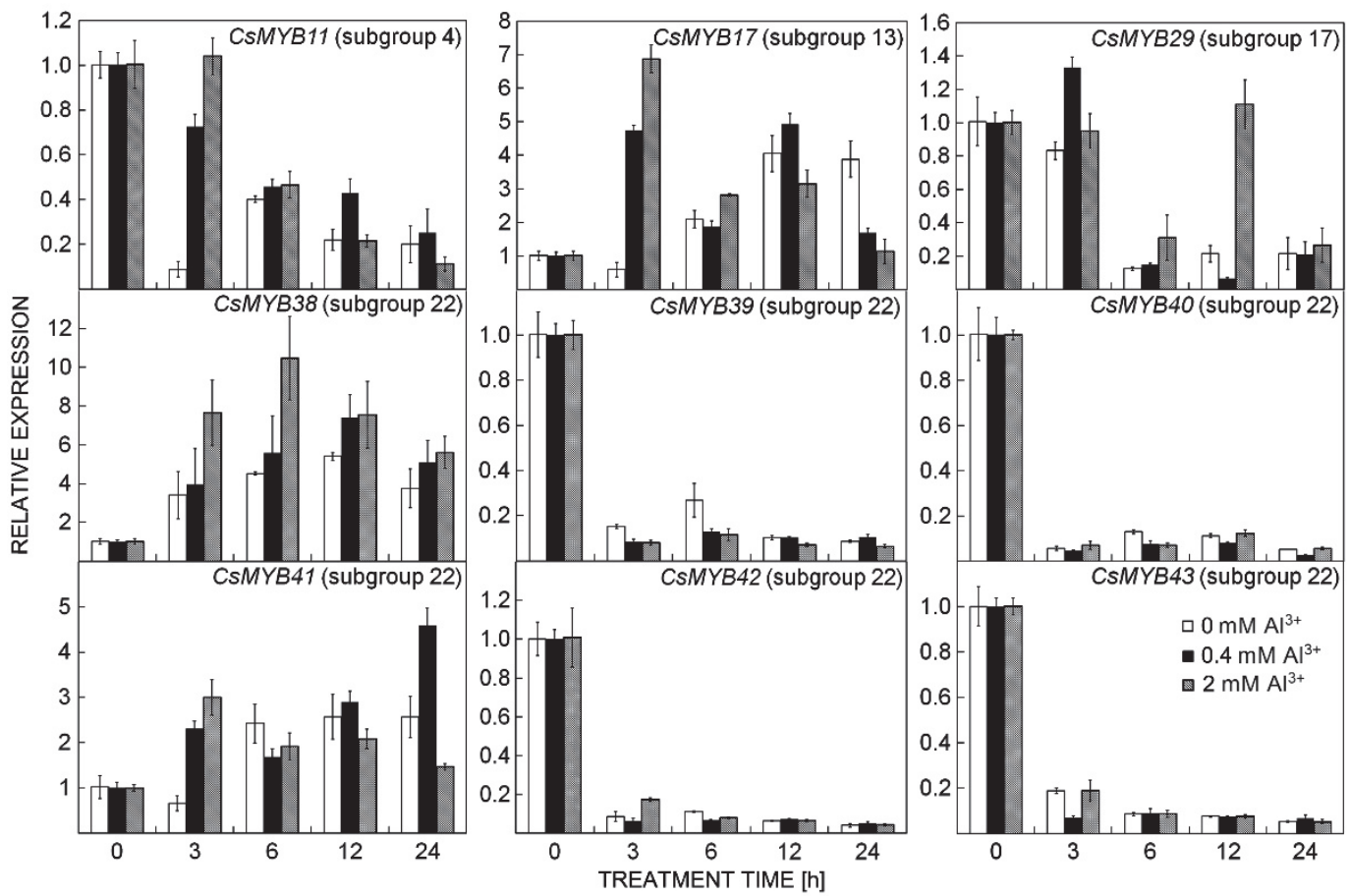

Fig. 5. The relative expression of 9 CsMYB genes at different $\mathrm{Al}^{3+}$ treatments $(0,0.4$, and $2 \mathrm{mM})$ for 0 to $24 \mathrm{~h}$. 
(subgroup 13) displayed a significant induction within $3 \mathrm{~h}$ at $2 \mathrm{mM} \mathrm{Al}^{3+}$, but the transcript accumulation declined to normal levels after $24 \mathrm{~h}$. Under $0.4 \mathrm{mM} \mathrm{Al}^{3+}$, the gene CsMYB29 (subgroup 17) was highly expressed within $3 \mathrm{~h}$, but had a low expression later. The CsMYB38 (subgroup 22) had the highest expression at $12 \mathrm{~h}$ under 0 and $0.4 \mathrm{mM}$ $\mathrm{Al}^{3+}$ and was efficiently induced also after $6 \mathrm{~h}$ under $2 \mathrm{mM}$ $\mathrm{Al}^{3+}$. Furthermore, the expression of CsMYB38 (subgroup 22) at $2 \mathrm{mM} \mathrm{Al}^{3+}$ for $6 \mathrm{~h}$ was higher than that at 0 and $0.4 \mathrm{mM} \mathrm{Al}^{3+}$ for $12 \mathrm{~h}$. The CsMYB41 (subgroup 22) was highly expressed at $24 \mathrm{~h}$ under $0.4 \mathrm{mM} \mathrm{Al}^{3+}$ treatment. Compared with the control groups, CsMYB39, CsMYB40, CsMYB42, CsMYB43 (subgroup 22) had similar expressions under $\mathrm{Al}^{3+}$ treatment and their expressions decreased from 3 to $24 \mathrm{~h}$ (Fig. 5).

Under the $\mathrm{F}^{-}$treatment (Fig. 6), it is apparently observed that CsMYB11 (subgroup 4), CsMYB17 (subgroup 13), CsMYB40, CsMYB42, and CsMYB43 (subgroup 22) have similar expressions under $8 \mathrm{mg}\left(\mathrm{F}^{-}\right) \mathrm{dm}^{-3}$. They all reached the high expressions at 3 $\mathrm{h}$ and their expressions further increased at $24 \mathrm{~h}$. The expression of CsMYB38 (subgroup 22) and CsMYB41 (subgroup 22) peaked at $6 \mathrm{~h}$ under $8 \mathrm{mg} \mathrm{dm}^{-3} \mathrm{~F}^{-}$. In addition, compared with the control group, CsMYB29 (subgroup 17) was observed to be down-regulated at $3 \mathrm{~h}$ and then increased at $6 \mathrm{~h}$ under $0 \mathrm{mg}\left(\mathrm{F}^{-}\right) \mathrm{dm}^{-3}$. The CsMYB17 (subgroup 13) was highly expressed at $0 \mathrm{mg}\left(\mathrm{F}^{-}\right) \mathrm{dm}^{-3}$ within $3 \mathrm{~h}$, but down-regulated later.
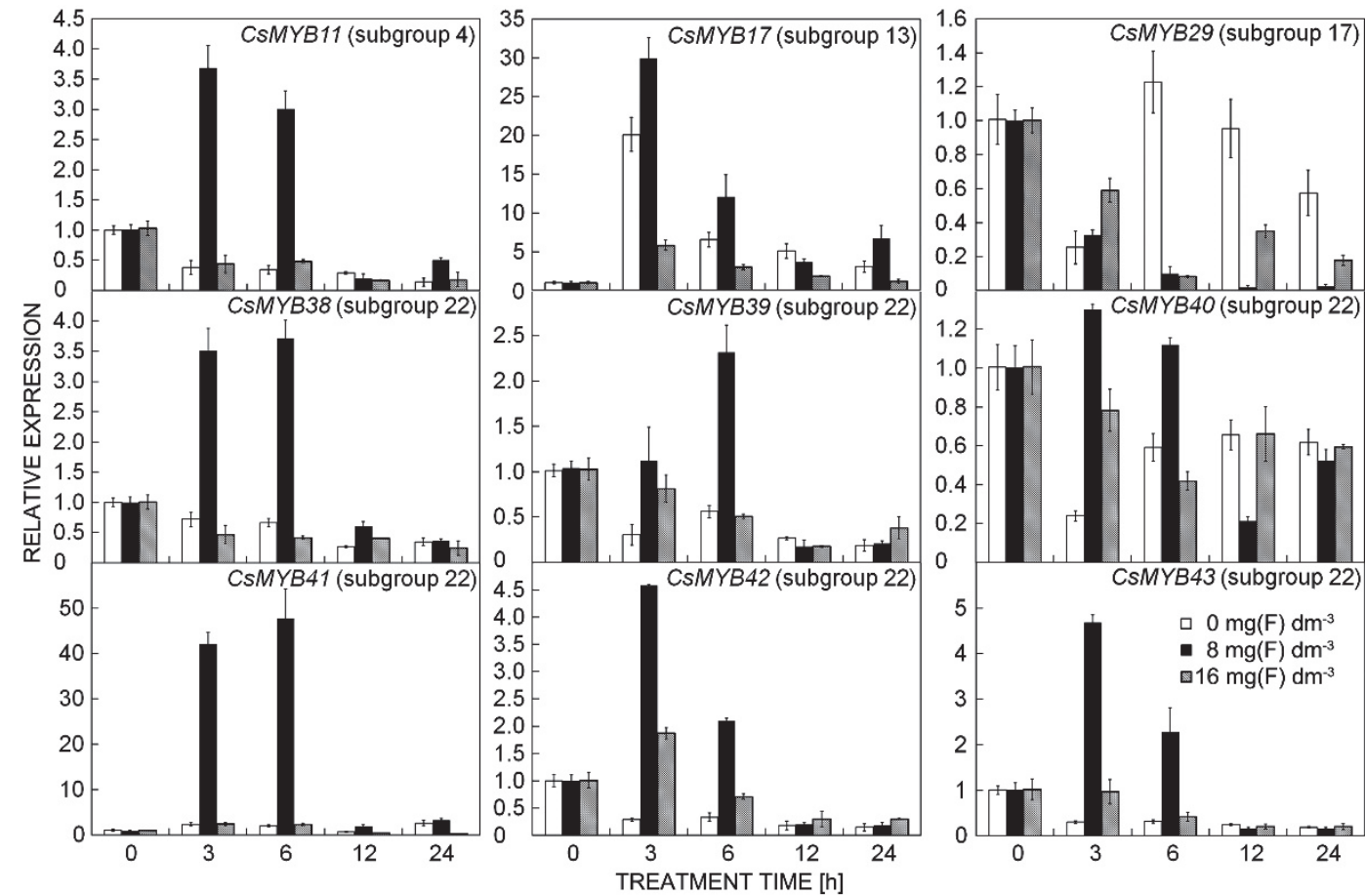

Fig. 6. The relative expression of 9 CsMYB genes at different $\mathrm{F}^{-}$treatments $\left(0,8\right.$, and $\left.16 \mathrm{mg} \mathrm{dm}^{-3}\right)$ for 0 to $24 \mathrm{~h}$.

\section{Discussion}

In previous studies, the R2R3-MYB TF has been identified in Arabidopsis (Dubos et al. 2010), in poplar (Wilkins et al. 2009), in rice (Katiyar et al. 2012), and in some other plants. Although individual R2R3-MYB genes have been identified in tea plant (Zhao et al. 2013, Sun et al. 2016), systematic analysis of the R2R3-MYB family has not been studied. Thus, we identified and analyzed $43 C s M Y B$ genes of this family in tea plant using transcriptome data and found that they all encode proteins with the R2R3-MYB domain. These $C s M Y B$ genes can be classified into 18 subgroups. According to the previous studies, we screened 9 CsMYB candidate genes that are related to abiotic stresses from $43 C s M Y B$ genes and used them for a further study. The relative expressions of these MYB genes in different tissues were analyzed for exploring their functions in C. sinensis.

We analyzed tissue-specific expression patterns of CsMYB genes (Fig. 4). The CsMYB29 (subgroup 17) showed the highest expression in roots and $\mathrm{Mu}$ et al. (2009) showed that AtMYB59, also belonging to subgroup 17 , regulates root development through the control of cell cycle progression at the root tips. Therefore, we predict that CsMYB29 might participate in the development of tea roots via control the root tip cell cycle progression. CsMYB38 to CsMYB43 were clustered in the same subgroup 22 (Fig. 1). According to the previous studies, 
AtMYB77 (subgroup 22) regulates lateral root formation by modulating the expression of auxin-inducible genes (Shin et al. 2007). In this study, CsMYB38 (subgroup 22) was also found to be highly expressed in the roots, indicating that $C s M Y B 38$ may participate in the growth of tea roots. In addition, AtMYB70 and AtMYB77 (subgroup 22) show up-regulation during stem development (Ko et al. 2004). CsMYB39 showed the highest expression in stems as well. CsMYB40 (subgroup 22) showed the highest expression in flowers, while CsMYB41 and CsMYB42 showed the highest expressions in pollen tubes and the expression of CsMYB43 was mostly observed in fruits and pollen tubes. The above results indicate that the various members of $C S M Y B$ (subgroup 22) might function in different tissues of $C$. sinensis.

The roots growth of $C$. sinensis is stimulated by $\mathrm{Al}^{3+}$ and $\mathrm{F}^{-}$(Fung et al. 2008, Zhang et al. 2013). In the present study, we detected the expression patterns of 9 CsMYB genes in $C$. sinensis roots after exposure to $\mathrm{Al}^{3+}$ and $\mathrm{F}^{-}$. Tea plant is known to be an $\mathrm{Al}^{3+}$ hyperaccumulator and the growth of roots is promoted at lower $\mathrm{Al}^{3+}$ concentrations but inhibited at high concentrations (Mukhopadyay et al. 2012). Comparing to control $\left(0 \mathrm{mM} \mathrm{Al}{ }^{3+}\right), C s M Y B 11$ (subgroup 4), CsMYB17 (subgroup 13) and CsMYB29 (subgroup 17) were up-regulated by $\mathrm{Al}$ treatment, suggesting that CsMYB11, -17 and -29 might influence root growth of $C$. sinensis in response to Al treatment. In addition, functional studies on R2R3-MYB revealed that R2R3-MYB genes in the same subgroup not only possess similar functions but also are co-expressed after stress treatments (Liu et al. 2010). For example, the AtMYB44, AtMYB70, AtMYB73, and AtMYB77 were classified into subgroup 22 and showed similar temporal and spatial expression pattern (Shin et al. 2007, Fan et al. 2014). Interestingly, $C s M Y B 38$ to $C s M Y B 43$ were grouped into subgroup 22 (Fig. 5). Compared with the control, CsMYB39, -40, -42 and -43 were all down-regulated by $\mathrm{Al}^{3+}$ treatments. It implies that the expression of CSMYB39, $-40,-42$ and -43 are negatively regulated by the uptake of $\mathrm{Al}^{3+}$. In addition, the CsMYB38 gene had the highest expression at $2 \mathrm{mM} \mathrm{Al}{ }^{3+}$. It is suggested that the $\mathrm{Al}^{3+}$ treatment could efficiently promote the expression of
CsMYB38 in tea roots. CsMYB41 was highly expressed at $0.4 \mathrm{mM} \mathrm{Al} l^{3+}$. The results implied that appropriate concentration of $\mathrm{Al}^{3+}$ could improve the expression of CsMYB41 (subgroup 22) in tea roots.

Some R2R3-MYB family members have been found to be related to the flavonoid biosynthesis and purple foliage coloration of tea plants (Zhao et al. 2013, Sun et al. 2016). However, the R2R3-MYB family involved in the process of absorption of $\mathrm{F}$ of tea plant has not yet been reported. In the present study, the expression of CsMYB11 (subgroup 4) and CsMYB17 (subgroup 13) peaked after $3 \mathrm{~h}$ at $8 \mathrm{mg}\left(\mathrm{F}^{-}\right) \mathrm{dm}^{-3}$. It is reasonable to speculate that the $8 \mathrm{mg}\left(\mathrm{F}^{-}\right) \mathrm{dm}^{-3}$ could efficiently improve the expression of CsMYB11 and CsMYB17. In addition, the functions of some Arabidopsis R2R3-MYB members, especially of the subgroup 22, have been well characterized experimentally and phylogenetically analyzed (Jung et al. 2008). In the present study, the relatively high expressions of CSMYB38, $-39,-40,-41,-42$, and -43 genes in response to the $8 \mathrm{mg}$ $\mathrm{dm}^{-3} \mathrm{~F}$ treatment suggest that these genes may somehow influence $\mathrm{F}^{-}$accumulation. In contrast, CsMYB29 (subgroup 17) gene expression is inhibited during exposure to $\mathrm{F}^{-}$. These data combined with the $C$. sinensis root growth patterns under different concentrations of $\mathrm{F}^{-}$ lead us to speculate that CsMYB11, -17, -38, -39, -40, -41, -42 and -43 are involved in $\mathrm{F}$ accumulation of $C$. sinensis roots.

In conclusion, $43 C s M Y B$ genes were identified for the first time in $C$. sinensis. We systematically analyzed their bioinformatic characteristics and found they can be classified into 18 subgroups. Furthermore, we select 9 CsMYB genes for exploring their expression patterns in different tissue and under $\mathrm{Al}$ and $\mathrm{F}$ stresses. These genes showed a diverse expression pattern in $C$. sinensis tissues. In addition, the expressions of $9 C S M Y B$ genes under $\mathrm{Al}^{3+}$ and $\mathrm{F}^{-}$stress indicate that $\operatorname{CsMYB11},-17,-38$, and -41 might function in the response to $\mathrm{Al}$ and $\mathrm{F}$ stresses and deserve further research. These findings are useful to expand our understanding of molecular responses to $\mathrm{Al}$ and $\mathrm{F}$ treatment in tea plant. Taken together, this study would provide a solid foundation for future functional investigation of the R2R3-MYB family in tea plant.

\section{References}

Cai, H.M., Dong, Y.Y., Li, Y.Y., Li, D.X., Peng, C.Y., Zhang, Z.Z., Wan, X.C.: Physiological and cellular responses to fluoride stress in tea (Camellia sinensis) leaves. - Acta Physiol. Plant. 38: 144. 2016.

Du, H., Feng, B.R., Yang, S.S., Huang, Y.B., Tang, Y.X.: The R2R3-MYB transcription factor gene family in maize. - Plos ONE 7: e37463, 2012a.

Du, H., Yang, S.S., Liang, Z., Feng, B.R., Liu, L., Huang, Y.B., Tang, Y.X.: Genome-wide analysis of the MYB transcription factor superfamily in soybean. - BMC Plant Biol. 12: 106, 2012b.
Dubos, C., Stracke, R., Grotewold, E., Weisshaar, B., Martin, C., Lepiniec, L.: MYB transcription factors in Arabidopsis. Trends Plant Sci. 15: 573-581, 2010.

Fan, J., Jiang, C., Xing, J., Dong, J.: Structure and function of the $22^{\text {nd }}$ subfamily in Arabidopsis R2R3-MYB family. Hereditas 36: 985-94, 2014.

Feng, C.P., Andreasson, E., Maslak, A., Mock, H.P., Mattsson, O., Mundy, J.: Arabidopsis MYB68 in development and responses to environmental cues. - Plant Sci. 167: 1099-1107, 2004.

Fung, K.F., Carr, H.P., Zhang, J., Wong, M.H.: Growth and 
nutrient uptake of tea under different aluminium concentrations. - J. Sci. Food Agr. 88: 1582-1591, 2008).

Fung, K.F., Zhang, Z.Q., Wong, J.W.C., Wong, M.H.: Fluoride contents in tea and soil from tea plantations and the release of fluoride into tea liquor during infusion. - Environ. Pollut. 104: 197-205, 1999.

Haga, N., Kato, K., Murase, M., Araki, S., Kubo, M., Demura, T., Suzuki, K., Muller, I., Voss, U., Jurgens, G., Ito, M.: R1R2R3-Myb proteins positively regulate cytokinesis through activation of KNOLLE transcription in Arabidopsis thaliana. - Development 134: 1101-1110, 2007.

Hajiboland, R., Bahrami, R.S., Barceló, J., Poschenrieder, C.: Mechanisms of aluminum-induced growth stimulation in tea (Camellia sinensis). - J. Soil Sci. Plant Nutr. 176: 616-625, 2013.

Ito, M.: Conservation and diversification of three-repeat Myb transcription factors in plants. - J. Plant Res. 118: 61-69, 2005.

Jung, C., Seo, J.S., Han, S.W., Koo, Y.J., Kim, C.H., Song, S.I., Nahm, B.H., Choi, Y.D., Cheong, J.J.: Overexpression of AtMYB44 enhances stomatal closure to confer abiotic stress tolerance in transgenic Arabidopsis. - Plant Physiol. 146: 623-35, 2008.

Katiyar, A., Smita, S., Lenka, S.K., Rajwanshi, R., Chinnusamy, V., Bansal, K.C.: Genome-wide classification and expression analysis of MYB transcription factor families in rice and Arabidopsis. - BMC Genomics 13: 544, 2012.

Ko, J.H., Han, K.H., Park, S., Yang, J.M.: Plant body weightinduced secondary growth in Arabidopsis and its transcription phenotype revealed by whole-transcriptome profiling. - Plant Physiol. 135: 1069-1083, 2004.

Li, Q.S., Lin, X.M., Qiao, R.Y., Zheng, X.Q., Lu, J.L., Ye, J.H., Liang, Y.R.: Effect of fluoride treatment on gene expression in tea plant (Camellia sinensis). - Sci. Rep. 7: 9847. 2017.

Li, Y., Huang, J., Song, X.W., Zhang, Z.W., Jiang, Y., Zhu, Y.L., Zhao, H., Ni, D.J.: An RNA-Seq transcriptome analysis revealing novel insights into aluminum tolerance and accumulation in tea plant. - Planta 246: 91-103, 2017.

Lippold, F., Sanchez, D.H., Musialak, M., Schlereth, A., Scheible, W.R., Hincha, D.K., Udvardi, M.K.: AtMyb41 regulates transcriptional and metabolic responses to osmotic stress in Arabidopsis. - Plant Physiol. 149: 1761-1772, 2009.

Liu, R.X., Lu, B.B., Wang, X.M., Zhang, C.L., Zhang, S.P., Qian, J., Chen, L., Shi, H.J., Dong, H.S.: Thirty-seven transcription factor genes differentially respond to a harpin protein and affect resistance to the green peach aphid in Arabidopsis. - J. Biosci. 35: 435-450, 2010.

Mu, R.L., Cao, Y.R., Liu, Y.F., Lei, G., Zou, H.F., Liao, Y., Wang, H.W., Zhang, W.K., Ma, B., Du, J.Z., Yuan, M., Zhang, J.S., Chen, S.Y.: An R2R3-type transcription factor gene AtMYB59 regulates root growth and cell cycle progression in Arabidopsis. - Cell Res. 19: 1291-1304, 2009.

Mukhopadyay, M., Bantawa, P., Das, A., Sarkar, B., Bera, B., Ghosh, P., Mondal, T.K.: Changes of growth, photosynthesis and alteration of leaf antioxidative defence system of tea [Camellia sinensis (L.) O. Kuntze] seedlings under aluminum stress. - Biometals 25: 1141-1154, 2012.

Noda, K., Glover, B.J., Linstead, P., Martin, C.: Flower color intensity depends on specialized cell-shape controlled by a Myb-related transcription factor. - Nature 369: 661-664, 1994.

Pan, J.T., Wang, W.D., Li, D.Q., Shu, Z.F., Ye, X.L., Chang, P.P., Wang, Y.H.: Gene expression profile indicates involvement of NO in Camellia sinensis pollen tube growth at low temperature. - BMC Genomics 17: 809, 2016.

Pfaffl, M.W.: A new mathematical model for relative quantification in real-time RT-PCR. - Nucl. Acids Res. 29, 2001.

Riechmann, J.L., Heard, J., Martin, G., Reuber, L., Jiang, C.Z., Keddie, J., Adam, L., Pineda, O., Ratcliffe, O.J., Samaha, R.R., Creelman, R., Pilgrim, M., Broun, P., Zhang, J.Z., Ghandehari, D., Sherman, B.K., Yu, C.L.: Arabidopsis transcription factors: genome-wide comparative analysis among eukaryotes. - Science 290: 2105-2110, 2000.

Ruan, J.Y., Wong, M.H.: Accumulation of fluoride and aluminium related to different varieties of tea plant. Environ. Geochem. Health. 23: 53-63, 2001.

Ruan, J.Y., Ma, L.F., Shi, Y.Z., Han, W.Y.: The impact of $\mathrm{pH}$ and calcium on the uptake of fluoride by tea plants (Camellia sinensis L.). - Ann. Bot. 93: 97-105. 2004.

Segarra, G., Van der Ent, S., Trillas, I., Pieterse, C.M.J.: MYB72, a node of convergence in induced systemic resistance triggered by a fungal and a bacterial beneficial microbe. Plant Biol. 11: 90-96, 2009.

Seo, P.J., Park, C.M.: Auxin homeostasis during lateral root development under drought condition. - Plant Signal Behav. 4: 1002-1004, 2009.

Shin, R., Burch, A.Y., Huppert, K.A., Tiwari, S.B., Murphy, A.S., Guilfoyle, T.J., Schachtman, D.P.: The Arabidopsis transcription factor MYB77 modulates auxin signal transduction. - Plant Cell 19: 2440-2453, 2007.

Stracke, R., Werber, M., Weisshaar, B.: The R2R3-MYB gene family in Arabidopsis thaliana. - Curr. Opin. Plant Biol. 4: 447-456, 2001.

Sun, B.M., Zhu, Z.S., Cao, P.R., Chen, H., Chen, C.M., Zhou, X., Mao, Y.H., Lei, J.J., Jiang, Y.P., Meng, W., Wang Y.X., Liu, S.Q.: Purple foliage coloration in tea (Camellia sinensis L.) arises from activation of the R2R3-MYB transcription factor CsAN1. - Sci. Rep. 6: 32534. 2016.

Tolra, R., Vogel-Mikus, K., Hajiboland, R., Kump, P., Pongrac, P., Kaulich, B., Gianoncelli, A., Babin, V., Barcelo, J., Regvar, M. Poschenrieder, C.: Localization of aluminium in tea (Camellia sinensis) leaves using low energy X-ray fluorescence spectro-microscopy. - J. Plant Res. 124:165$172,2011$.

Wilkins, O., Nahal, H., Foong, J., Provart, N.J., Campbell, M.M.: Expansion and diversification of the Populus R2R3-MYB family of transcription factors. - Plant Physiol. 149: 981-993, 2009.

Wong, M.H., Zhang, Z.Q., Wong, J.W.C., Lan, C.Y.: Trace metal contents $(\mathrm{Al}, \mathrm{Cu}$ and $\mathrm{Zn}$ ) of tea: tea and soil from two tea plantations, and tea products from different provinces of China. - Environ. Geochem. Health. 20: 87-94, 1998.

Xie, Z.D., Li, D.M., Wang, L.J., Sack, F.D., Grotewold, E.: Role of the stomatal development regulators FLP/MYB88 in abiotic stress responses. - Plant J. 64: 731-739, 2010.

Xu, Q.S., Wang, Y., Ding, Z.T., Fan, K., Ma, D.X., Zhang, Y.L., Yin, Q.: Aluminum induced physiological and proteomic responses in tea (Camellia sinensis) roots and leaves. - Plant Physiol. Biochem. 115: 141-151, 2017.

Xu, Q., Wang, Y., Ding, Z., Song, L., Li, Y., Ma, D., Wang, Y., Shen, J., Jia, S., Sun, H. Zhang, H.: Aluminum induced metabolic responses in two tea cultivars. - Plant Physiol. Biochem. 101: 162-172. 2016.

Yang, Y., Liu, Y., Huang, C.F., De Silva, J., Zhao, F.J.: Aluminium alleviates fluoride toxicity in tea (Camellia 
sinensis). - Plant Soil 402:179-190. 2016.

Zhang, L., Li, Q., Ma, L.F., Ruan, J.Y.: Characterization of fluoride uptake by roots of tea plants (Camellia sinensis (L.) O. Kuntze). - Plant Soil 366: 659-669, 2013.

Zhao, L., Gao, L.P., Wang, H.X., Chen, X.T., Wang, Y.S., Yang, H., Wei, C.L., Wan, X.C., Xia, T.: The R2R3-MYB, bHLH,
WD40, and related transcription factors in flavonoid biosynthesis. - Funct. integr. Genomics 13: 75-98, 2013.

Zhou, C.P., Chen, Y.B., Wu, Z.Y., Lu, W.J., Han, J.L., Wu, P.Z., Chen, Y.P., Li, M.R., Jiang, H.W., Wu, G.J.: Genome-wide analysis of the MYB gene family in physic nut (Jatropha curcas L.). - Gene 572: 63-71, 2015. 